\title{
KRULL DIMENSION OF MODULES AND INVOLUTIVE IDEALS
}

\author{
S. C. COUTINHO
}

(Communicated by Ken Goodearl)

\begin{abstract}
In this paper we establish an upper bound for the Krull dimension of a module over a Weyl algebra in terms of a geometrical invariant of its characteristic variety, the involutive dimension. This is followed by some examples which show that this inequality may be strict.
\end{abstract}

\section{INTRODUCTION}

In this paper we show that the Krull dimension of a module over the Weyl algebra is related to geometrical invariants of the characteristic variety of that module. We begin with a survey of a few basic facts about modules over the Weyl algebra and their characteristic varieties.

Let $A_{n}$ denote the $n$th Weyl algebra over the field of complex numbers $\mathbb{C}$. The generators of the algebra $A_{n}$ will be denoted by $x_{i}$ and $\partial_{i}=\partial / \partial x_{i}$ for $1 \leq i \leq n$. We endow $A_{n}$ with the Bernstein filtration, obtained by giving each $x_{i}$ and each $\partial_{i}$ degree one. The $k$ th step in this filtration will be denoted by $A_{n}(k)$. We write $S_{n}$ for the graded ring of $A_{n}$ with respect to the Bernstein filtration and $S_{n}(k)$ for its $k$ th homogeneous component. The symbol of order $k$ is the canonical map $\sigma_{k}: A_{n}(k) \rightarrow A_{n}(k) / A_{n}(k-1)=S_{n}(k)$. Thus $S_{n}$ is a polynomial ring in the $2 n$ variables $y_{1}, \ldots, y_{2 n}$, where $y_{i}=\sigma_{1}\left(x_{i}\right)$ and $y_{i+n}=\sigma_{1}\left(\partial_{i}\right)$ for $1 \leq i \leq n$.

Let $\{$,$\} denote the Poisson bracket of S_{n}$ associated to the standard symplectic structure of $\mathbb{C}^{2 n}$. The Poisson bracket is a bilinear and skew symmetric operation in $S_{n}$ which satisfies the Leibniz rule: if $f, g, h \in S_{n}$, then $\{f, g h\}=g\{f, h\}+h\{f, g\}$. It may be used to calculate the symbol of a bracket of two operators in $A_{n}$ as follows: If $d \in A_{n}(k)$ and $d^{\prime} \in A_{n}(m)$, then $\sigma_{k+m-2}\left(\left[d, d^{\prime}\right]\right)=\left\{\sigma_{k}(d), \sigma_{m}\left(d^{\prime}\right)\right\}$. For more details see [1] or [10].

Let $M$ be a finitely generated left $A_{n}$-module and $F$ a good filtration of $M$. By that we mean a filtration of $M$ for which $\mathrm{gr}^{F} M$ is finitely generated over $S_{n}$. Let $I(M)$ be the radical of the annihilator of $\mathrm{gr}^{F} M$ in $S_{n}$. Note that $I(M)$ is a homogeneous ideal of $S_{n}$. The ideal $I(M)$ is called the characteristic ideal of $M$ and its variety $\operatorname{char}(M)$ in $\mathbb{C}^{2 n}$, the characteristic variety of $M$. Both $I(M)$ and $\operatorname{char}(M)$ are independent of the choice of the good filtration

Received by the editors May 19, 1993 and, in revised form, September 24, 1993. 1991 Mathematics Subject Classification. Primary 16S32, 16P60, 16W50. 
used to define them and hence, are invariants of $M$. The dimension $d(M)$ of $\operatorname{char}(M)$ equals the Gelfand-Kirillov dimension of $M$.

The ideal $I(M)$ is an involutive ideal of $S_{n}$ with respect to the Poisson bracket defined above, namely, $\{I(M), I(M)\} \subseteq I(M)$. Equivalently, the variety $\operatorname{char}(M)$ is involutive in the sense that its tangent spaces are co-isotropic for the standard skew-symmetric form in $\mathbb{C}^{2 n}$; see [1]. The involutivity of $I(M)$ was proved by Gabber in [3] using purely algebraic methods. An important consequence of the involutivity of $I(M)$ is the inequality $d(M) \geq n$.

Let us now review some of the results that relate invariants of the characteristic variety and the Krull dimension of $A_{n}$-modules. Let $M$ be a finitely generated left $A_{n}$-module, with $\operatorname{Krull}$ dimension $\operatorname{Kdim}(M)$. It follows from [8, Corollary 8.5.6] that $d(M) \geq \operatorname{Kdim}(M)+n$. Björk had conjectured in [2] that the equality holds in the formula above. This, however, is false. Stafford showed in [9] that there exist simple $A_{n}$-modules of Gelfand-Kirillov dimension $2 n-1$; his example is also discussed in [5, Chapter 8].

Following a suggestion of J. T. Stafford we consider in this paper another formula for the Krull dimension of modules over the Weyl algebra. This is inspired by results of Bernstein and Lunts in [1] and [7]. For a finitely generated left $A_{n}$-module $M$, let $\beta(M)$ be the length of the longest chain of involutive homogeneous irreducible subvarieties contained in $\operatorname{char}(M)$. We prove in $\S 2$ that $\operatorname{Kdim}(M) \leq \beta(M)$ and that $d(M) \geq \beta(M)+n$. However, both these inequalities may be strict, as shown by the examples of $\S 3$.

\section{INVOLUTIVE DIMENSION}

We begin with the definition of a new dimension for ideals of $S_{n}$. Let $I$ be a homogeneous ideal of $S_{n}$. The involutive dimension of $I$, denoted by $\beta(I)$, is the length of the longest chain of homogeneous involutive prime ideals containing $I$. Let $M$ be a finitely generated left $A_{n}$-module. It is convenient to define the involutive dimension of $M$, denoted by $\beta(M)$, to be the involutive dimension of $I(M)$; namely $\beta(M)=\beta(I(M))$. This is equivalent to the definition in terms of $\operatorname{char}(M)$ given in the introduction. We proceed to state some of the properties of the involutive dimension. Our first lemma is a wellknown result; we include a proof for the sake of completeness.

Lemma 2.1. Let I be a homogeneous involutive radical ideal of $S_{n}$. The primes minimal over I are homogeneous involutive prime ideals of $S_{n}$.

Proof. Let $P$ be a prime ideal minimal over $I$. It is easy to show that $P$ must be homogeneous; we show that it is involutive. Let $a, a^{\prime} \in P$; we shall prove that $\left\{a, a^{\prime}\right\} \in P$. Let $J$ be the intersection of the prime ideals minimal over $I$ and different from $P$. Let $b \in J \backslash P$. Since $J P \subseteq I$, we must have that $\left\{a b, a^{\prime} b\right\} \in I \subseteq P$, but $\left\{a b, a^{\prime} b\right\}=\left\{a b, a^{\prime}\right\} b+\{a b, b\} a^{\prime}$. Thus $\left\{a b, a^{\prime}\right\} b \in P$. Since $P$ is prime and $b \notin P$, one has that $\left\{a b, a^{\prime}\right\} \in P$. Once again, $\left\{a b, a^{\prime}\right\}=\left\{a, a^{\prime}\right\} b+\left\{b, a^{\prime}\right\} a$, from which we conclude that $\left\{a, a^{\prime}\right\} \in P$, as required.

Recall that the coheight of an ideal $I$ of $S_{n}$ is the length of the longest chain of prime ideals of $S_{n}$ that contain $I$. It will be denoted by $\operatorname{coht}(I)$.

Lemma 2.2. If $I$ is an involutive ideal of $S_{n}$, then $\beta(I) \leq \operatorname{coht}(I)-n$. 
Proof. Let $P_{0} \subset P_{1} \subset \cdots \subset P_{s}$ be the longest chain of involutive homogeneous prime ideals containing $I$. Then $\operatorname{coht}(I) \geq \beta(I)+\operatorname{coht}\left(P_{s}\right)$. Since $P_{s}$ is an involutive ideal, $\operatorname{coht}\left(P_{s}\right) \geq n$. Hence $\operatorname{coht}(I) \geq \beta(I)+n$.

Let $J$ be a left ideal of $A_{n}$. The symbol ideal of $J$ is

$$
\sigma(J)=\sum_{k \geq 0}\left(\sigma_{k}\left(J \cap A_{n}(k)\right) .\right.
$$

It is an ideal of $S_{n}$. The next result collects some of the properties of the involutive dimension. In particular it follows from Proposition 2.3(b) that $\beta$ is an exact dimension function in the sense of $[8,6.8 .4]$. On the other hand, Proposition $2.3(\mathrm{~d})$ is a generalisation of [1, Theorem A].

Proposition 2.3. The involutive dimension of a finitely generated left $A_{n}$-module $M$ satisfies

(a) $\beta(M)=\max \{\beta(P), P$ a prime minimal over $I(M)\}$.

(b) If $0 \rightarrow M^{\prime} \rightarrow M \rightarrow M^{\prime \prime} \rightarrow 0$ is an exact sequence of finitely generated $A_{n}$-modules, then $\beta(M)=\max \left\{\beta\left(M^{\prime}\right), \beta\left(M^{\prime \prime}\right)\right\}$.

(c) $d(M) \geq \beta(M)+n$.

(d) If $J$ is a left ideal of $A_{n}$ such that $\sigma(J)$ is prime and $\beta(\sigma(J))=0$, then $J$ is a maximal left ideal of $A_{n}$. In other words, $A_{n} / J$ is a simple left $A_{n}$-module.

Proof. Note that (a) follows from Lemma 2.1 and the fact that $I(M)$ is a radical involutive ideal of $S_{n}$ whilst (c) follows from Lemma 2.2. To prove (b), note that $I(M)=I\left(M^{\prime}\right) \cap I\left(M^{\prime \prime}\right)$. Thus, if $P$ is a prime ideal of $S_{n}$, then it contains $I(M)$ if and only if it contains either $I\left(M^{\prime}\right)$ or $I\left(M^{\prime \prime}\right)$. The equality follows immediately from this and (a).

Finally, we prove (d). Suppose that $L$ is a left ideal of $A_{n}$ that properly contains $J$. Thus $\sigma(J) \subset \sigma(L) \subseteq S_{n}$. Taking radicals and recalling that $\sigma(J)$ is prime, one obtains the proper inclusion, $\sigma(J) \subset \operatorname{rad}(\sigma(L)) . \operatorname{But} \operatorname{rad}(\sigma(L))=$ $I\left(A_{n} / L\right)$ is an involutive homogeneous radical ideal of $S_{n}$. Since $\beta(\sigma(J))=0$, we must have that $\beta(\operatorname{rad}(\sigma(L)))=0$. But $\sigma(J)$ is prime and the inequality $\sigma(J) \subset \operatorname{rad}(\sigma(L))$ is proper. Hence $\operatorname{rad}(\sigma(L))=S_{n}$ and $L=A_{n}$.

Now we shall prepare the ground for the proof that the involutive dimension is an upper bound for the Krull dimension. The main step in the proof of this result is Theorem 2.6. Its proof makes use of the multiplicity of an $A_{n}$ module at a prime ideal of $S_{n}$, which we briefly describe. Let $M$ be a finitely generated left $A_{n}$-module with a good filtration $F$ and $P$ a prime ideal of $S_{n}$. The multiplicity $m_{P}(M)$ of $M$ with respect to $P$ is the length of the $\left(S_{n}\right)_{P-}$ module $\left(\operatorname{gr}^{F} M\right)_{P}$. The multiplicity thus defined is independent of the good filtration $F$ used to calculate it and is additive over short exact sequences of $A_{n}$-modules. The multiplicity also satisfies the following property: $m_{P}(M)$ is finite and nonzero if and only if $M \neq 0$ and $P$ is a prime ideal minimal over $I(M)$. For details see [1, $\S 3]$ and [6, Chapter V, Proposition 2.7]. In the proof of the theorem we use that, under certain circumstances, the length of a descending chain of $A_{n}$-modules is bounded by the multiplicity. This is the content of the following lemma. We omit the proof, as it is easy and very similar to [5, Corollary 7.8]. 
Lemma 2.4. Let $M$ be a finitely generated left $A_{n}$-module and $P$ a prime minimal over $I(M)$. Suppose that $M=M_{0} \supset M_{1} \supset \cdots \supset M_{s}$ is a strictly descending chain of submodules of $M$ for which $P$ is minimal over $I\left(M_{i} / M_{i+1}\right)$ for $i=0,1,2, \ldots$. Then

(a) $m_{P}\left(M / M_{i}\right)=\sum_{j=0}^{i-1} m_{P}\left(M_{j} / M_{j+1}\right)$,

(b) $s \leq m_{P}(M)$.

One more technical lemma is necessary before we come to the proof of the theorem.

Lemma 2.5. Let $M$ be a finitely generated left $A_{n}$-module and $N$ a submodule of $M$. If $P$ is a homogeneous prime ideal of $S_{n}$ minimal over $I(N)$ and $P$ does not contain $I(M / N)$, then $P$ is minimal over $I(M)$.

Proof. Since $I(M)=I(N) \cap I(M / N)$, it follows that $P$ contains $I(M)$. Suppose that $P$ is not minimal over $I(M)$. Thus there exists a homogeneous prime ideal $Q$ such that $I(M) \subseteq Q \subset P$. In particular $I(N) \cdot I(M / N) \subseteq Q$. Since $I(M / N)$ is not contained in $P$, we must have that $I(N) \subseteq Q$. But this contradicts the fact that $P$ is minimal over $I(N)$.

We want to show that the Krull dimension of a module is bounded above by its involutive dimension. Following [8, Chapter 8], it is enough to prove that $A_{n}$ is finitely partitive for $\beta$. Recall that $A_{n}$ is (left) finitely partitive for $\beta$ if given any finitely generated left $A_{n}$-module $M$, then every chain $M=M_{0} \supseteq M_{1} \supseteq \cdots$ of submodules of $M$, such that $\beta\left(M_{i} / M_{i+1}\right)=\beta(M)$ for all $i \geq 0$, must terminate. This is the weaker definition of finitely partitive, proposed in [8, 8.7.3].

Theorem 2.6. $A_{n}$ is finitely partitive for the involutive dimension.

Proof. Let $M$ be a finitely generated left $A_{n}$-module. Suppose that $M=M_{0} \supseteq$ $M_{1} \supseteq \cdots$ is a chain of submodules of $M$ such that $\beta\left(M_{i} / M_{i+1}\right)=\beta(M)$ for $i=0,1,2, \ldots$.

Let $\mathbb{P}$ be the (finite) set of homogeneous involutive prime ideals minimal over $I(M)$. Given $i \in \mathbb{N}$ we have that $I\left(M_{i} / M_{i+1}\right) \supseteq I(M)$ and $\beta\left(M_{i} / M_{i+1}\right)=$ $\beta(M)$; thus at least one prime ideal in $\mathbb{P}$ must be minimal over $I\left(M_{i} / M_{i+1}\right)$. Since $\mathbb{P}$ is finite, there exists a prime $P \in \mathbb{P}$ which is minimal over infinitely many of the ideals $I\left(M_{i} / M_{i+1}\right)$.

Now if $P$ is minimal over $I\left(M_{i} / M_{i+1}\right)$ but not over $I\left(M_{i-1} / M_{i}\right)$, then by Lemma 2.5 it is minimal over $I\left(M_{i-1} / M_{i+1}\right)$. Thus by deleting from the sequence $M=M_{0} \supset M_{1} \supset \cdots$ the terms $M_{i}$ whenever $P$ is not minimal over $I\left(M_{i-1} / M_{i}\right)$, we obtain an infinite subsequence

$$
M=N_{0} \supset N_{1} \supset N_{2} \supset \cdots
$$

for which $P$ is minimal over all $I\left(N_{i} / N_{i+1}\right)$. But this contradicts Lemma 2.4 and completes the proof of the theorem.

Corollary 2.7. The involutive dimension of a finitely generated left $A_{n}$-module $M$ satisfies:

(a) $d(M) \geq \beta(M)+n \geq \operatorname{Kdim}(M)+n$.

(b) If $\beta(M)=0$, then $M$ is a module of finite length. 
Proof. The first inequality in (a) follows from Proposition 2.3(c). The second inequality follows from the fact that $A_{n}$ is finitely positive for $\beta$ (see $[8,8.7 .3$ and 8.3.18], whilst (b) follows from (a).

Note that the inequalities above may be strict. This is illustrated in the next section, where several examples are discussed. It is perhaps worth observing that most of the above can be generalized to almost commutative rings.

\section{EXAMPLES}

We now discuss some examples for which the inequalities of the previous section are strict. Consider first $d(M) \geq \beta(M)+n$. Bernstein and Lunts showed in [1,7] that for most operators $d \in A_{n}, n \geq 2$, the module $M=A_{n} / A_{n} d$ satisfies $\beta(M)=0$. Since $d(M)=2 n-1$, the inequality is strict in this case.

We now turn to $\operatorname{Kdim}(M) \leq \beta(M)$. Let $d=\partial_{1}+\left(1+\lambda x_{1} x_{2}\right) \partial_{2}+x_{2}$. Stafford showed in [9] that the module $M=A_{2} / A_{2} d$ is simple. Thus $\operatorname{Kdim}(M)=0$. But $I(M)$ is the ideal generated by $y_{1} y_{2} y_{4}$ in $S_{2}$. Hence $\left(y_{4}\right)$ is a prime ideal minimal over $I(M)$. But $\left(y_{4}\right) \subseteq\left(y_{3}, y_{4}\right)$ and $\left(y_{3}, y_{4}\right)$ is involutive. Hence, $\beta(M)=1$, and we conclude that $\operatorname{Kdim}(M)<\beta(M)$, in this case.

The characteristic ideal of $M$, in this last example, is not prime. However, even when the characteristic ideal is prime, the inequality $\operatorname{Kdim}(M) \leq \beta(M)$ may be strict. To construct such an example, we shall use a result of Bernstein and Lunts [1]. Let $V_{k}$ be the space of vector fields on $\mathbb{C}^{2}$ of the form $P_{1} \partial_{1}+$ $P_{2} \partial_{2}$, where $P_{1}$ and $P_{2}$ are polynomials of degree $\leq k-2$ in $x_{1}, x_{2}$. For a vector field $\xi$ in $\mathbb{C}^{2}$ and a singular point $s$ of $\xi$, let $\Lambda(\xi, s)$ denote the subgroup of $\mathbb{C}$ generated by the eigenvalues of the linear operator on $T_{s} \mathbb{C}^{2}$, defined by the one-jet of $\xi$.

Given a vector field $\xi=P_{1} \partial_{1}+P_{2} \partial_{2}$, let $Y_{\xi}$ be the hypersurface $P_{1}\left(y_{1}, y_{2}\right) y_{3}$ $+P_{2}\left(y_{1}, y_{2}\right) y_{4}=0$ of $\mathbb{C}^{4}$. Thus $Y_{\xi}$ is involutive with respect to the standard symplectic structure of $\mathbb{C}^{4}$. It is also conical, that is, homogeneous with respect to the variables $y_{3}$ and $y_{4}$. We say that $Y_{\xi}$ is minimal if the only involutive conical variety contained in $Y_{\xi}$ are of the following type: $Z\left(y_{3}, y_{4}\right)$ or $Z\left(y_{1}-\alpha_{1}, y_{2}-\alpha_{2}\right)$, for a point $\left(\alpha_{1}, \alpha_{2}\right) \in \mathbb{C}^{2}$. In other words, $\mathbb{C}^{4}$ is the cotangent bundle of $\mathbb{C}^{2}$, and $Y_{\xi}$ is minimal if its only involutive conical subvarieties are the zero section or fibres of this bundle.

Theorem 3.1. Let $k \geq 4$ and $\xi \in V_{k}$. Suppose that $Y_{\xi}$ is minimal and that $\xi$ has a finite, nonempty set of singular points in $\mathbb{C}^{2}$. Choose a polynomial $f \in \mathbb{C}\left[x_{1}, x_{2}\right]$ such that, for each singular point $s \in \mathbb{C}^{2}, f(s) \notin \Lambda(\xi, s)$. Then the operator $\xi+f$ generates a maximal left ideal of $A_{2}$.

Before this theorem is put to use, we must obtain vector fields $\xi$ for which $Y_{\xi}$ is minimal. First of all, we endow $V_{k}$ with the topology of affine space. The natural structure follows from the fact that $V_{k}$ is a complex vector space with basis $x_{1}^{r} x_{2}^{s} \partial_{i}$, where $r+s \leq k-2$ and $i=1,2$. The corresponding coordinate functions will be denoted by $z_{r s i}$. We say that a property $\Sigma$ holds generically in $V_{k}$ if the set $\left\{\xi \in V_{k}: \Sigma\right.$ does not hold for $\left.\xi\right\}$ is contained in the union of a countable number of hypersurfaces in $V_{k}$. The next result is a consequence of [1, Theorem 5].

Theorem 3.2. The property " $Y_{\xi}$ is minimal" holds generically in $V_{k}$ for $k \geq 4$. 
The example is constructed as follows.

Theorem 3.3. Let $4 \leq k \leq 7$ be an integer. There exists $d \in A_{2}$ which satisfies:

(a) $A_{2} d$ is a maximal left ideal of $A_{2}$.

(b) $d$ has degree $m=k-1$ for the Bernstein filtration, and the hypersurface $\sigma_{m}(d)=0$ in $\mathbb{P}^{3}(\mathbb{C})$ is an irreducible homogeneous subvariety of $\mathbb{P}^{3}(\mathbb{C})$.

(c) $\sigma_{m}(d)$ is contained in the ideal generated by $y_{1}$ and $y_{2}$ in $S_{2}$.

In particular, $A_{2} / A_{2} d$ is a simple module, but $I\left(A_{2} / A_{2} d\right)$ is prime and has involutive dimension 1 .

It is better to isolate part of the proof in a lemma. If $P \in \mathbb{C}\left[x_{1}, x_{2}\right]$, denote by $P^{k}$ the homogeneous component of degree $k$ of $P$.

Lemma 3.4. Suppose that $k \geq 4$. Let $X$ be the set of all vector fields $P_{1} \partial_{1}+P_{2} \partial_{2}$ in $V_{k}$ such that at least one of the three conditions below is satisfied:

(I) $P_{1}^{k-2} \cdot P_{2}^{k-2}=0$.

(II) $P_{1}$ and $P_{2}$ have a common factor.

(III) $P_{1}^{k-2}$ and $P_{2}^{k-2}$ have a common factor.

Then $X$ is contained in a union of hypersurfaces of $V_{k}$.

Proof. Let $\xi=P_{1} \partial_{1}+P_{2} \partial_{2}$ be a vector field in $X$. If $\xi$ satisfies (I), then it is contained in the union of the hypersurfaces of equations $z_{k-2,0, i}=0$ for $i=1,2$.

Suppose that $P_{1}, P_{2}$ have a factor in common. We may assume that $z_{k-2,0, i}$ $\neq 0$ for $i=1,2$. Thus $P_{1}$ and $P_{2}$ are monic as polynomials in $x_{1}$. If they have a common factor in $\mathbb{C}\left[x_{1}, x_{2}\right]$, then they have a common factor in $\mathbb{C}\left(x_{2}\right)\left[x_{1}\right]$. Hence the resultant $\operatorname{Res}_{x_{1}}\left(P_{1}, P_{2}\right)$, with respect to $x_{1}$, is identically zero. The coefficients of this resultant with respect to $x_{2}$ are polynomials in $z_{r s i}$. Equating one of these coefficients to zero we have a hypersurface that contains $\xi$. Thus $\xi$ is contained in a union of hypersurfaces when (II) holds. One may deal with (III) in a similar way.

Proof of Theorem 3.3. By Theorem 3.2 and Lemma 3.4, there exists $\xi \in V_{k}$ such that $Y_{\xi}$ is minimal and (I), (II), and (III) are not satisfied by $\xi$. Let $S_{\xi}$ stand for the set of singular points of $\xi$. We show that $S_{\xi}$ is finite and nonempty. Let $\xi=P_{1} \partial_{1}+P_{2} \partial_{2}$, where $P_{1}, P_{2}$ are polynomials of degree $\leq k-2$.

A singularity of $\xi$ is a zero of the system $P_{1}=P_{2}=0$. Write $W_{1}$ and $W_{2}$ for the curves of $\mathbb{P}^{2}(\mathbb{C})$ that correspond to the sets of zeros of the homogenizations of $P_{1}$ and $P_{2}$, respectively. Then $W_{1} \cap W_{2} \neq \varnothing$. Let $w \in W_{1} \cap W_{2}$. If $w$ belongs to the line at infinity, then its homogeneous coordinates are $[\alpha: \beta$ : 0]. Hence $(\alpha, \beta)$ is a zero of $P_{1}^{k-2}$ and $P_{2}^{k-2}$. Since these polynomials are homogeneous, this implies that they have a common factor, contradicting (III). Thus $w$ must be a finite point, and so $w \in S_{\xi} \neq \varnothing$.

Since $P_{1}$ and $P_{2}$ do not have a common factor, we may apply Bezout's Theorem and conclude that $S_{\xi}$ contains at most $(k-2)^{2}$ elements. We will use this to show that there exists a polynomial $Q \in \mathbb{C}\left[x_{1}, x_{2}\right]$ of degree $\leq k-1$ which has the elements of $S_{\xi}$ for zeros. This is the place in the proof where we need to have that $k \leq 7$.

Let $U(k)$ be the vector space of polynomials in $\mathbb{C}\left[x_{1}, x_{2}\right]$ of degree $\leq k-1$. Put $S_{\xi}=\left\{s_{1}, \ldots, s_{n}\right\}$. Consider the linear mapping $\phi: U(k) \rightarrow \mathbb{C}^{n}$ defined by $\phi(Q)=\left(Q\left(s_{1}\right), \ldots, Q\left(s_{n}\right)\right)$. Since $\operatorname{dim} U(k)=\left(\begin{array}{c}1+k \\ 2\end{array}\right)=\left(k^{2}+k\right) / 2$, it follows 
that $\operatorname{dim} U(k)>(k-2)^{2} \geq n$ for $k \geq 7$. Thus $\phi$ has a nonzero kernel. Hence there exists $0 \neq Q \in U(k)$ such that $Q(s)=0$ for every $s \in S_{\xi}$.

Now let $L \subseteq \mathbb{C}$ denote the union of the $\Lambda(\xi, s)$ for every $s \in S_{\xi}$. There are at most $2 \cdot(k-2)^{2}$ such eigenvalues. Choose a complex number $\alpha$ algebraically independent with the set $L$. Put $f=Q+\alpha$. For each $s \in S_{\xi}$ one has that $f(s)=Q(s)+\alpha=\alpha \notin \Lambda(\xi, s)$. Thus we may apply Theorem 3.1 to the operator $d=P_{1} \partial_{1}+P_{2} \partial_{2}+f$ and conclude that the left ideal $A_{2} d$ is maximal. Hence $(a)$ is verified.

Let $G=\sigma_{k-1}(d)=P_{1}^{k-2}\left(y_{1}, y_{2}\right) y_{3}+P_{2}^{k-2}\left(y_{1}, y_{2}\right) y_{4}+Q^{k-1}\left(y_{1}, y_{2}\right)$. Note that $G$ is linear in $y_{3}, y_{4}$. If it were reducible, then at least one of its factors would be a nonconstant polynomial in $y_{1}, y_{2}$. But this is not possible, because $P_{1}^{k-2}$ and $P_{2}^{k-2}$ have no common factor. Thus $G$ must be irreducible homogeneous polynomial. Therefore, the ideal $(G)$ in $S_{2}$ is prime, and $G=0$ defines an irreducible hypersurface in $\mathbb{P}^{3}(\mathbb{C})$.

That $G \subseteq\left(y_{1}, y_{2}\right)$ is immediate; thus (c) is also verified. Since $\left(y_{1}, y_{2}\right)$ is obviously prime and involutive, it follows that $\beta\left(A_{2} / A_{2} d\right) \geq 1$. Thus, by Corollary 2.7, $\beta\left(A_{2} / A_{2} d\right)=1$, and the proof is complete.

The fact that the inequality $\operatorname{Kdim}(M) \leq \beta(M)$ may be strict admits a geometrical interpretation. It is closely related to the breakdown of the following extension property for left ideals of $A_{n}$. Let $J$ be a left ideal of $A_{n}$. Suppose that $P$ is a homogeneous prime ideal of height 1 over $\operatorname{rad}(\sigma(J))$. Is there a left ideal $L$ of $A_{n}$ such that $J \subseteq L$ and $P$ is minimal over $\operatorname{rad}(\sigma(L))$ ? A counterexample to this extension property is provided by the ideal $A_{n} d$ of Theorem 3.3. In fact, if $J=A_{n} d$, then $\operatorname{rad}(\sigma(J))=(G)$ is a prime ideal. The prime ideal $\left(y_{1}, y_{2}\right)$ has height 2 and contains $(G)$, which has height 1 . Hence $\left(y_{1}, y_{2}\right)$ has height 1 over $(G)$. But $J=A_{n} d$ is maximal; hence the extension property must fail in this case.

Let us end with a question related to the above extension property, the answer to which does not seem to be known. Let $J$ be a radical involutive homogeneous ideal of $S_{n}$. Is there a finitely generated left $A_{n}$-module $M$ such that $I(M)=$ $J$ ? On this question, see also [10, Chapter I, Theorem 6.21] and [2, Chapter 4, Theorem 6.5].

\section{ACKNOWLEDGMENTS}

I began work on this paper after conversations with J. T. Stafford during the London Mathematical Society Symposium on "Noncommutative rings: new directions" in July 1992. I wish to thank Stafford for bringing the problem to my attention, the organisers for an exciting and valuable conference, and M. P. Holland and the referee for suggestions and corrections on a previous version of this paper.

\section{REFERENCES}

1. J. Bernstein and V. Lunts, On non-holonomic irreducible D-modules, Invent. Math. 94 (1988), 223-243.

2. J.-E. Björk, Rings of differential operators, North-Holland, Amsterdam, 1979.

3. O. Gabber, The integrability of the characteristic variety, Amer. J. Math. 103 (1981), 445-465.

4. R. Hatshorne, Algebraic geometry, Springer-Verlag, New York and Berlin, 1977. 
5. G. R. Krause and T. Lenagan, Growth of algebras and Gelfand-Kirillov dimension, Pitman, London, 1985.

6. E. Kunz, Introduction to commutative algebra and algebraic geometry, Birkhäuser, Boston, 1985.

7. V. Lunts, Algebraic varieties preserved by generic flows, Duke Math. J. 58 (1989), 531-554.

8. J. C. McConnell and J. C. Robson, Noncommutative noetherian rings, Wiley, London, 1988.

9. J. T. Stafford, Non-holonomic modules over Weyl algebras and enveloping algebras, Invent. Math. 79 (1985), 619-638.

10. P. Schapira, Microdifferential systems in the complex domain, Springer-Verlag, Berlin and Heidelberg, 1985.

Instituto de Matemática, Universidade Federal do Rio de Janeiro, C. Postal 68.530, 21 945-970 RIO RJ, BRAZIL

E-mail address: collier@impa.br 